\title{
Theoretical understanding of the increment of $\beta$ upon protonation of pyridine peripheral octupolar molecules: Toward nonlinear optical sensors
}

\author{
M. Carmen Ruiz Delgado \\ School of Chemistry and Biochemistry, Georgia Institute of Technology, Atlanta, Georgia 30332-0400, USA \\ and Department of Organic Chemistry, ICMA, University of Zaragoza-CSIC, Zaragoza 50009, Spain \\ Jesús Orduna \\ Department of Organic Chemistry, ICMA, University of Zaragoza-CSIC, Zaragoza 50009, Spain \\ Maria Moreno Oliva, Juan Casado, and Juan T. López Navarrete ${ }^{a}$ \\ Department of Physical Chemistry, University of Málaga, Campus de Teatinos s/n, Málaga 29071, Spain
}

(Received 27 July 2007; accepted 18 September 2007; published online 23 October 2007)

In this article, we present a computational study of the nonlinear optical properties of pyridine-based octupolar molecules in their neutral and fully triprotonated states. The effect of the core substitution and the degree of conjugation with the periphery has been also established and confirms the possibility of fine-tuning the nonlinear optical response. Computations involving the time-dependent density-functional theory approach serve to further explore the existence of excited states with nonzero dipole moment. From these results, the origin of the high second-order nonlinear optical activity upon protonation is addressed. (C) 2007 American Institute of Physics.

[DOI: $10.1063 / 1.2796167]$

\section{INTRODUCTION}

The design and development of molecular nonlinear optical (NLO) materials with high nonlinear optical activity have attracted considerable scientific interest because of their potential applications in emerging optoelectronic and alloptical data processing technologies. ${ }^{1,2}$ Although the field of NLO organic molecules is dominated by the donor-acceptor substituted dipolar molecules, it has become apparent that the dipoles have certain limitations (i.e., difficult noncentrosymmetric crystallization in the solid state to achieve a maximum bulk effect). After the recognition that octupolar systems can circumvent these disadvantages, there has been intense research directed to the synthesis and optimization of their NLO response. ${ }^{3-5}$ More recently, there is a growing interest in the design of functional electro-optical molecules able to act simultaneously as sensing systems (i.e., whose NLO properties can be modulated by electrochemical or chemical inputs such as protons or metal cations). ${ }^{6}$ Nevertheless, to the best of our knowledge, there is only very few reports on octupolar NLO systems with switchable secondorder nonlinearity. ${ }^{7}$ Theoretical calculations on octupolar trisacetylene benzenes suggest that the second-order hyperpolarizability $(\beta)$ could increase more than one order of magnitude upon introduction of positive charges at the pyridyl end groups in comparison with the neutral species. These considerations lead Hennrich et al. to design octupolar chomophores based on core benzenes trigonally ramified with acetylene-pyridinium branches (Fig. 1) with protontriggered NLO response. ${ }^{9}$ In this paper, we report an evaluation of the NLO properties of this family of octupolar 1,3,5trisalkynylbenzenes derivates by using quantum chemistry

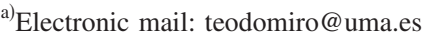

tools in order to elucidate the impact of the chemical pattern modifications (i.e., core substitution and peripheral conjugation) on their electronic structure (i.e., core-to-branches or branches-to-branches $\pi$-conjugational coupling). The origin of the great enhancement in the second-order NLO activity upon protonation has also been rationalized on the basis of the nature of the degenerate excited states by time-dependent density-functional theory calculations.

\section{COMPUTATIONAL METHOD}

Density-functional theory (DFT) calculations were carried out by means of the GAUSSIAN03 program ${ }^{10}$ running on a SGI Origin 2000 supercomputer. The molecular geometries were optimized assuming $C_{3 h}$ symmetry by using the Becke's three-parameter exchange functional combined with the LYP correlation functional ${ }^{11}$ (B3LYP) and the standard $6-31 \mathrm{G}^{* *}$ basis set. ${ }^{12}$ Optimal geometries were determined on isolated entities in the vacuum. Vertical electronic excitation energies were computed by using the time-dependent DFT (TD-DFT) approach ${ }^{13,14}$ and excited state dipole moments were calculated using the RhoCI density on the previously optimized molecular geometries. Molecular orbital contours were plotted by using MOLEKEL $4.3{ }^{15}$

The tensor components of the static first hyperpolarizability have been analytically calculated by using the coupled perturbed Hartree-Fock (CPHF) method. ${ }^{16,17}$ Zyss et al. have developed and discussed the tensorial nature of the first hyperpolarizability for octupolar molecules. ${ }^{3,18-20}$ Within this framework, the first hyperpolarizability tensor can be decomposed into two components $\beta_{J=1}$ and $\beta_{J=3}$, the dipolar and octupolar contributions,

$$
\beta=\beta_{J=1} \otimes \beta_{J=3},
$$




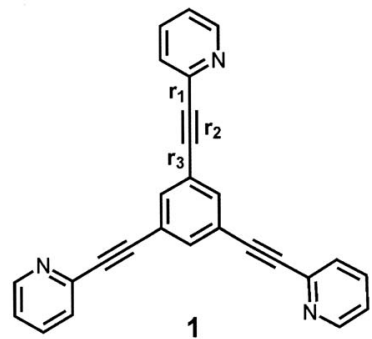

1

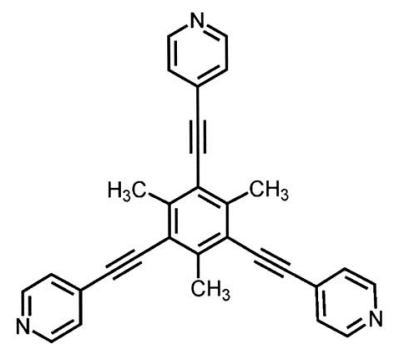

3

FIG. 1. Chemical structures of 1,3,5-tris(pyridylethynyl)benzene derivates $1-3$.

$$
\|\beta\|^{2}=\left\|\beta_{J=1}\right\|^{2}+\left\|\beta_{J=3}\right\|^{2} .
$$

For two-dimensional (2D) planar system with no further symmetric assumptions, the following expressions may be applicable:

$$
\begin{aligned}
& \left\|\beta_{J=1}^{2 D}\right\|^{2}=\frac{3}{4}\left[\left(\beta_{X X X}+\beta_{X Y Y}\right)^{2}+\left(\beta_{Y Y Y}+\beta_{Y X X}\right)^{2}\right], \\
& \left\|\beta_{J=3}^{2 D}\right\|^{2}=\frac{1}{4}\left[\left(\beta_{X X X}-3 \beta_{X Y Y}\right)^{2}+\left(\beta_{Y Y Y}-3 \beta_{Y X X}\right)^{2}\right] .
\end{aligned}
$$

\section{RESULTS AND DISCUSSION}

\section{A. First hyperpolarizabilities: CPHF calculations}

Making use of theoretical calculations, we have determined that $C_{3 h}$ conformation in which the three pyridylethynyl units are oriented in the same fashion and are coplanar to the central benzene ring is the most stable structure adopted for these octupolar 1,3,5-trisalkynylbenzenes derivates. This feature is supported by ${ }^{13} \mathrm{C}$ NMR data. ${ }^{9}$ Therefore, we have considered that the octupolar trispyridyl compounds present planar structures and we assume the 2D approach on the determination of each irreducible tensor components of $\beta$.
Moreover, it has been recently demonstrated for different octupolar triazines that the effects of the deviations from the planarity on $\beta$ are rather small. ${ }^{21}$

Table I presents the calculated values of the first hyperpolarizabilties and their tensor elements for molecules 1-3 and their protonated forms $1^{+3}-3^{+3}$. These tensor components for molecules belonging to the $C_{3 h}$ group approximately have the following relationships:

$$
\begin{aligned}
& \beta_{x x x}=-\beta_{x y y}, \\
& \beta_{y y y}=-\beta_{y x x} .
\end{aligned}
$$

As a consequence, pyridine-based octupolar molecules may present an isotropic $\beta$ tensor, for which the off-diagonal coefficients can be derived from simple symmetry arguments. Comparing the first and last column of Table I, we observe that the theoretical and the experimental hyperpolarizabilities determined by hyper-Rayleigh scattering (HRS) are in excellent agreement, having in mind that the $\beta$ values have been calculated on the lowest-energy conformations in the vacuum and experimental data were obtained in solution. The $\pi$-electron density of the benzene core may act as a donor relative to the peripheral $s p$ hybridized $\mathrm{C} \equiv \mathrm{C}$ carbon atoms; thus, upon inclusion of hyperconjugating donor substituents $\left(-\mathrm{CH}_{3}\right)$, more favorable core-to-branches charge transfer is expected which would enhance the $\beta$ values. This suggestion becomes obvious when comparing the experimental $\beta$ values in Table I on passing from compound 1 to the mesityl derivatives 2 and 3 both in neutral and triply charged states. This experimental trend is also well reproduced by theoretical calculations and is in good concordance with the continuous downshift displacement of the $\nu(\mathrm{C} \equiv \mathrm{C})$ Raman band observed on 1-3, which has been diagnosed to be sensitive to the increment of $\pi$-electron delocalization. $^{22}$

Let us now inspect the influence of the charge-transfer (CT) character not only from the pyridinium acceptor units toward the central benzene core but also between the different branches of the molecules on the NLO response. To this end, the octupole properties are compared to those of dipolar analogs. We have made use of a tensorial interaction framework introduced by Andrau et al. to evaluate the specific contribution of the octupolar symmetry to $\beta .^{23}$ In this additive model, ${ }^{23}$ generally applicable to multipolar systems, the $\beta$ tensor is dissected into two separate tensor representations, an additive term $\beta^{A}$ representing the tensorial sum of the

TABLE I. First hyperpolarizabilities and their tensor elements of optimized geometries of octupolar compounds assuming the case of a $2 \mathrm{D}$ planar system calculated by CPHF method using 6-31G** basis set. (All of the values are given in $10^{-30}$ esu.)

\begin{tabular}{ccccccc}
\hline \hline Molecules & $\beta_{x x x}$ & $\beta_{x y y}$ & $\beta_{y y y}$ & $\beta_{y x x}$ & $\|\beta\|$ & $\beta_{x x x 0}{ }^{\mathrm{a}}$ \\
\hline 1 & 3.606 & -3.606 & 0.518 & -0.518 & 7.29 & $8 \pm 1$ \\
$1^{+3}$ & 15.929 & -15.929 & 0.613 & -0.613 & 31.88 & $19 \pm 3$ \\
2 & 10.368 & -10.368 & -0.029 & -0.029 & 20.736 & $9 \pm 1$ \\
$2^{+3}$ & 26.305 & -26.305 & 3.1294 & -3.1294 & 52.98 & $38 \pm 2$ \\
3 & 11.634 & -11.634 & 1.122 & -1.122 & 23.37 & $12 \pm 3$ \\
$3^{+3}$ & 45.34 & -45.34 & 3.552 & -3.552 & 90.96 & $146 \pm 5$ \\
\hline \hline
\end{tabular}

${ }^{\mathrm{a}} \mathrm{HRS}$ data obtained in THF- $\mathrm{HCl}_{\mathrm{aq}}$ from Ref. 9. 


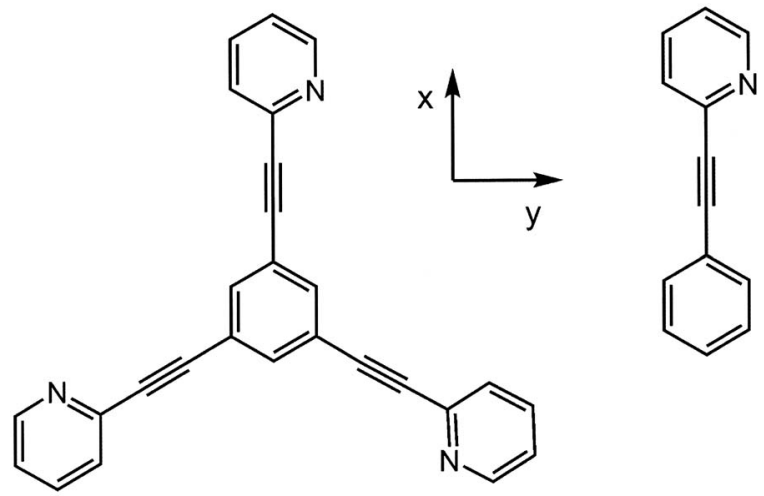

FIG. 2. Orientation of compound 1 taken as a prototype of the pyridinebased octupolar compounds. Its parent dipole unit as a reference system is also drawn.

dipole units $\beta$ and a term $\beta^{J}$ representing interactions between the dipole units constituting the multipole:

$$
\begin{aligned}
& \beta=\beta^{A} \otimes \beta^{J}, \\
& \beta^{A}=\sum_{i} \beta^{(i)} .
\end{aligned}
$$

The tensor $\beta^{(i)}$ of each dipole unit may be decomposed into dipolar and octupolar components $\beta_{J=1}^{(i)}$ and $\beta_{J=3}^{(i)}$ :

$$
\beta^{A}=\beta_{J=1}^{A} \otimes \beta_{J=3}^{A}=\sum_{i} \beta_{J=1}^{(i)} \otimes \sum_{i} \beta_{J=3}^{(i)} .
$$

The octupolar molecules may be defined as three independent one-dimensional (1D) units such as displayed in Fig. 2. Thus, Eq. (8) can be rewritten as Eq. (9) where $\beta_{J=1}^{1 \mathrm{D}}$ and $\beta_{J=3}^{1 \mathrm{D}}$ represent the dipolar and octupolar contributions of the $1 \mathrm{D}$ unit, respectively, and $\theta=120^{\circ}$ (Ref. 19):

$$
\begin{aligned}
\beta^{A} & =\left(\beta_{J=1}^{1 \mathrm{D}} \otimes \beta_{J=3}^{1 \mathrm{D}}\right)(1+\cos 3 \theta+\cos 6 \theta) \\
& =3\left(\beta_{J=1}^{1 \mathrm{D}} \otimes \beta_{J=3}^{1 \mathrm{D}}\right) .
\end{aligned}
$$

From Eqs. (2)-(4), respectively, and for the case of a $2 \mathrm{D}$ planar system the norm of a tensor $\beta$ and its dipolar and octupolar components $\beta_{J=1}$ and $\beta_{J=3}$ can be calculated. Thus the norm of $\beta^{A}$ can be defined by the following expression:

$$
\left\|\beta^{A}\right\|^{2}=3\left(\beta_{X X X}^{1 \mathrm{D}}\right)^{2}+3\left(\beta_{Y Y Y}^{1 \mathrm{D}}\right)^{2}+9\left(\beta_{X Y Y}^{1 \mathrm{D}}\right)^{2}+9\left(\beta_{Y X X}^{1 \mathrm{D}}\right)^{2} .
$$

According to Eq. (6), the interaction term $\beta^{I}$ can now be obtained from the difference between the tensor $\beta$ and $\beta^{A}$; thus, its norm is expressed by Eq. (11):

$$
\left\|\beta^{I}\right\|^{2}=\|\beta\|^{2}-\left\|\beta^{A}\right\|^{2}=\left\|\beta_{J=1}^{2 \mathrm{D}}\right\|^{2}+\left\|\beta_{J=3}^{2 \mathrm{D}}\right\|^{2}-\left\|\beta^{A}\right\|^{2} .
$$

The norms of $\beta^{A}$ and $\beta^{I}$ are calculated by using $\mathrm{CPHF} / 6-31 \mathrm{G}^{* *}$ for the optimized geometries of the octupo-
TABLE III. Calculated $\|\beta\|$ values CPHF/6-31G ${ }^{* *}$, HOMO-LUMO energy gap $(\Delta E)$, and BLA values of $1-3$ and $1^{+3}-3^{+3}$.

\begin{tabular}{cccc}
\hline \hline Molecules & $\|\beta\|^{\mathrm{a}}$ & $\mathrm{BLA}^{\mathrm{b}}$ & $\Delta E^{\mathrm{c}}$ \\
\hline 1 & 7.29 & 0.213 & 4.232 \\
$1^{+3}$ & 31.88 & 0.204 & 3.907 \\
2 & 20.736 & 0.212 & 4.111 \\
$2^{+3}$ & 52.98 & 0.202 & 3.702 \\
3 & 23.37 & 0.209 & 4.151 \\
$3^{+3}$ & 90.96 & 0.199 & 3.505 \\
\hline$\overline{\mathrm{a}} 10^{-30}$ esu. & & \\
${ }^{\mathrm{b}}(\mathrm{r} 1+\mathrm{r} 3) / 2-\mathrm{r} 2$, see Fig. 1. & & \\
${ }^{\mathrm{c}} \Delta E=E_{\text {LUMO }}-E_{\text {Hомо }}$ in eV. & &
\end{tabular}

lar molecules 1-3 according to Eqs. (10) and (11) (see Table II). The main results shown in Table II are as follows: (i) the order of $\left\|\beta^{I}\right\|$ is the same as that of $\left\|\beta^{A}\right\|$; (ii) calculated $\beta$ values for the 1D dipole units of molecules 1-3 increase in the following order: $3>2>1$. This result corroborates that the increase order of the $\beta$ values for the octupolar compounds is attributed to the $\mathrm{CH}_{3}$-substituted benzene core and its degree of conjugation with the periphery; (iii) the electron donor strength of the core and the orto-/para-substitution pattern of the $\mathrm{N}$ atoms in the periphery not only enhance the norm $\left\|\beta^{A}\right\|$ of the additive term but also the norm $\left\|\beta^{I}\right\|$ of the interaction term, suggesting an increase of the CT character from the central core to the periphery and also among the different branches of the molecules. It must be stressed that the additive model is not applicable to the triply charged compounds $1^{+3}-3^{+3}$ because the monoprotonated dipolar units have a very high dipole moment (i.e., the protonation of the dipolar subunit of 1 resulted in an increase of the dipole moment from $1.69 \mathrm{D}$ in the neutral form to $8.72 \mathrm{D}$ for the monocharged 1D unit). Thus, the additive term $\beta^{A}$ is overestimated and the monoprotonated dipolar unit is not a good representation of the subunit in the triply charged octupolar system.

\section{B. Structure-NLO properties relationship}

Table III compares the calculated $\|\beta\|$ values of compounds $1-3$ and $1^{+3}-3^{+3}$ with respect to the bond-length alternation (BLA) and the highest occupied molecular orbitallowest unoccupled molecular orbital (HOMO-LUMO) energy gap $(\Delta E)$. BLA has been calculated by subtracting the triple bond length of the conjugation bridge from the average length of the two $\mathrm{C}-\mathrm{C}$ bonds connected to it. The generation of charged pyridinium units upon protonation decreases the lengths of the single $\mathrm{CC}$ bonds $(r 1, r 3)$ and increases the length of the triple $\mathrm{CC}$ bond $(r 2)$, see Fig. 1 for the numerical bonding. Consequently, the calculated BLA decreases

TABLE II. First hyperpolarizabilities $\left\|\beta^{\mathrm{A}}\right\|$ and $\left\|\beta^{\mathrm{I}}\right\|$ for octupolar compounds $1-3$ calculated by CPHF method using $6-31 \mathrm{G}^{* *}$ basis set. (All of the values are given in $10^{-30} \mathrm{esu}$.)

\begin{tabular}{cllllll}
\hline \hline Molecules & $\beta_{x x x}^{1 \mathrm{D}}$ & $\beta_{x y y}^{1 \mathrm{D}}$ & $\beta_{y y y}^{1 \mathrm{D}}$ & $\beta_{y x x}^{1 \mathrm{D}}$ & $\left\|\beta^{\mathrm{A}}\right\|$ & $\left\|\beta^{\mathrm{I}}\right\|$ \\
\hline 1 & 2.721 & 0 & 0 & 0 & 4.71 & 5.557 \\
2 & 5.180 & 0.030 & 0.164 & 0.145 & 8.98 & 18.83 \\
3 & 5.724 & -0.047 & 0.024 & -0.172 & 9.918 & 21.128 \\
\hline \hline
\end{tabular}




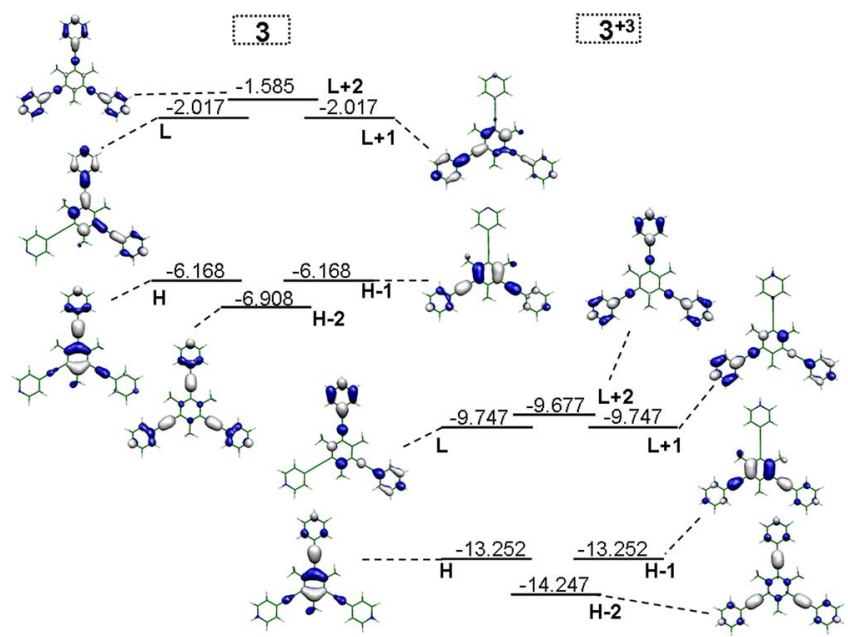

FIG. 3. (Color online) Molecular orbital diagram showing the electron density contours $\left(0.035 e\right.$ bohr $\left.^{3}\right)$ calculated for the frontier molecular orbitals of octupolar molecules 3 and $3^{+3}$.

upon generation of the triply charged octupolar molecule. In the same way, $\Delta E$ becomes smaller from the neutral to the protonated form. It is also observed that BLA and $\Delta E$ decrease upon replacement of benzene by mesitylene and after orto $\rightarrow$ para change on the pyridyl groups. Interestingly, as seen in Table III, $\beta$ values increase as BLA and $\Delta E$ decrease. This result agrees with the previous conclusion that $\beta$ values of octupolar molecules become larger as BLA decreases. ${ }^{24,25}$

\section{Nature of excited states: TD-DFT calculations}

To rationalize the enhancement in the second-order NLO activity upon protonation in a more intuitive way, we present in this section a study of the contribution of the different excited states (dipolar or octupolar) on the quadratic NLO response by means of TD-DFT calculations. The analysis of DFT molecular orbital (MO) diagram reveals the presence of pairs of degenerate orbitals which do not display an octupolar symmetry (i.e., $C_{3 h}$ ), whereas the nondegenerate orbitals (i.e., HOMO-2 and LUMO+2) do show (see Figs. 3 and 4).

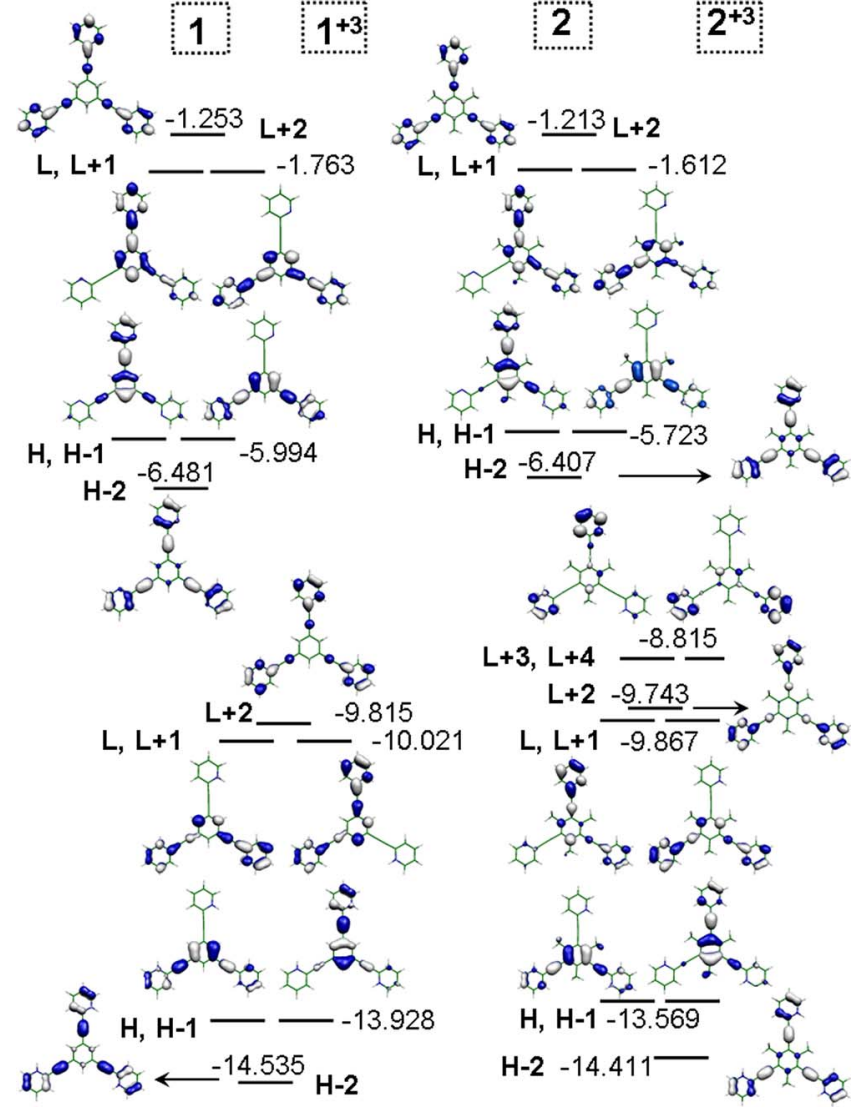

FIG. 4. (Color online) Molecular orbital diagram showing the electron density contours $\left(0.035 e \mathrm{bohr}^{3}\right)$ calculated for the frontier molecular orbitals of 1 and 2 and their protonated counterparts $1^{+3}$ and $2^{+3}$.

The existence of those pairs of degenerate orbitals without trigonal symmetry can give rise to localized degenerate excited states. $^{23,24,26}$

In an attempt to break the orbital degeneracy associated with $C_{3 h}$, symmetry, TD-DFT calculations were also performed on slightly distorted geometries having only $C_{1}$ symmetry. The resulting orbitals, while no degenerate, were nearly identical in energy to those obtained from symmetri-

TABLE IV. Results of TD-B3LYP/6-31G** calculations on the 1,3,5-trisalkynylbenzene derivates 3 and $3^{+3}$.

\begin{tabular}{|c|c|c|c|c|c|}
\hline Molecules & $\begin{array}{l}\mathrm{E}_{\max } \\
(\mathrm{eV})\end{array}$ & $f_{\text {osc }}{ }^{\mathrm{a}}$ & Major contributions $^{\mathrm{b}}$ & $\begin{array}{c}\left|\mu_{12}\right| \\
\text { (D) }\end{array}$ & $\begin{array}{c}\left|\Delta \mu_{12}\right| \\
\text { (D) }\end{array}$ \\
\hline \multirow[t]{8}{*}{3} & 3.85 & 1.05 & $\mathrm{H}-1 \rightarrow \mathrm{L}+1(42 \%)$ & 8.34 & 0.99 \\
\hline & & & $\mathrm{H} \rightarrow \mathrm{L}(42 \%)$ & & \\
\hline & 3.85 & 1.05 & $\mathrm{H}-1 \rightarrow \mathrm{L}(42 \%)$ & 8.34 & 0.99 \\
\hline & & & $\mathrm{H} \rightarrow \mathrm{L}+1(-42 \%)$ & & \\
\hline & 4.15 & 0.21 & $\mathrm{H} \rightarrow \mathrm{L}+2(91 \%)$ & 3.57 & 8.24 \\
\hline & 4.15 & 0.21 & $\mathrm{H}-1 \rightarrow \mathrm{L}+2(91 \%)$ & 3.57 & 8.24 \\
\hline & 4.42 & 0.22 & $\mathrm{H}-2 \rightarrow \mathrm{L}(90 \%)$ & 3.64 & 8.52 \\
\hline & 4.42 & 0.22 & $\mathrm{H}-2 \rightarrow \mathrm{L}+1(90 \%)$ & 3.64 & 8.52 \\
\hline \multirow[t]{4}{*}{$3^{+3}$} & 3.21 & 1.07 & $\mathrm{H} \rightarrow \mathrm{L}+2(56 \%)$ & 9.21 & 9.15 \\
\hline & 3.21 & 1.07 & $\mathrm{H}-1 \rightarrow \mathrm{L}+2(56 \%)$ & 9.21 & 9.15 \\
\hline & 4.08 & 0.24 & $\mathrm{H}-2 \rightarrow \mathrm{L}+1(85 \%)$ & 3.71 & 11.32 \\
\hline & 4.08 & 0.24 & $\mathrm{H}-2 \rightarrow \mathrm{L}+1(85 \%)$ & 3.71 & 11.32 \\
\hline
\end{tabular}


TABLE V. Results of TD-B3LYP/6-31G** calculations on neutral octupolar compounds 1 and 2.

\begin{tabular}{|c|c|c|c|c|}
\hline Molecules & $\mathrm{E}_{\max }(\mathrm{eV})$ & $f_{\text {osc }}{ }^{\mathrm{a}}$ & Major contributions $^{\mathrm{b}}$ & $\begin{array}{r}\left|\mu_{12}\right| \\
\text { (D) }\end{array}$ \\
\hline \multirow[t]{8}{*}{1} & 3.91 & 1.11 & $\mathrm{H}-1 \rightarrow \mathrm{L}(39 \%)$ & 8.64 \\
\hline & & & $\mathrm{H} \rightarrow \mathrm{L}+1(39 \%)$ & \\
\hline & 3.91 & 1.11 & $\mathrm{H}-1 \rightarrow \mathrm{L}+1(-39 \%)$ & 8.64 \\
\hline & & & $\mathrm{H} \rightarrow \mathrm{L}(39 \%)$ & \\
\hline & 4.26 & 0.14 & $\mathrm{H}-2 \rightarrow \mathrm{L}(90 \%)$ & 2.92 \\
\hline & 4.26 & 0.14 & $\mathrm{H}-2 \rightarrow \mathrm{L}+1(90 \%)$ & 2.92 \\
\hline & 4.32 & 0.31 & $\mathrm{H} \rightarrow \mathrm{L}+2(88 \%)$ & 4.36 \\
\hline & 4.32 & 0.31 & $\mathrm{H}-1 \rightarrow \mathrm{L}+2(88 \%)$ & 4.36 \\
\hline \multirow[t]{8}{*}{2} & 3.81 & 1.09 & $\mathrm{H}-1 \rightarrow \mathrm{L}(-43 \%)$ & 7.99 \\
\hline & & & $\mathrm{H} \rightarrow \mathrm{L}+1(43 \%)$ & \\
\hline & 3.81 & 1.09 & $\mathrm{H}-1 \rightarrow \mathrm{L}+1(-40 \%)$ & 7.99 \\
\hline & & & $\mathrm{H} \rightarrow \mathrm{L}(40 \%)$ & \\
\hline & 4.08 & 0.21 & $\mathrm{H} \rightarrow \mathrm{L}+2(92 \%)$ & 3.63 \\
\hline & 4.08 & 0.21 & $\mathrm{H}-1 \rightarrow \mathrm{L}+2(92 \%)$ & 3.63 \\
\hline & 4.34 & 0.22 & $\mathrm{H}-2 \rightarrow \mathrm{L}(73 \%)$ & 3.67 \\
\hline & 4.34 & 0.22 & $\mathrm{H}-2 \rightarrow \mathrm{L}+1(73 \%)$ & 3.67 \\
\hline
\end{tabular}

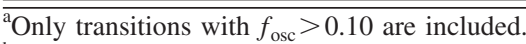

${ }^{\mathrm{b}} \mathrm{H}=\mathrm{HOMO}, \mathrm{L}=\mathrm{LUMO}$; only contributions above $20 \%$ are included.

cal structures and gave rise to excited states with nonzero dipole moments and therefore are not reported.

The description of the main electronic transitions for the doubly degenerate states of 3 and $3^{+3}$ is sketched on Table IV. For the neutral form 3 , the degenerate excited states with largest dipole moment change are associated with the highest-energy degenerate electronic transitions calculated at $4.15 \mathrm{eV}(f=0.21)$ and $4.42 \mathrm{eV}(f=0.22)$, whereas those associated with the lowest-energy degenerate electronic transitions related to the intense absorption maxima experimentally observed at $313 \mathrm{~nm}$ (Ref. 22) have a small dipole moment change $(0.99 \mathrm{D})$. It is worth noting that the transitions from the ground state to the degenerate excited states in one of those pairs are polarized orthogonally, and therefore, the transition to each excited state contributes to a different component of $\beta\left(\beta_{x x x}\right.$ or $\left.\beta_{y x x}\right)$. Calculations on the neutral compounds 1 and 2 lead to an analogous picture regarding that described for 3 (Fig. 4 and Table V).

By contrast, upon generation of the triply charged compound $3^{+3}$, the dipole moment change associated with the lowest-energy degenerate electronic transitions calculated at $3.21 \mathrm{eV}(f=1.07)$ and related to the broad and unstructured absorption band measured at $330 \mathrm{~nm}$ (Ref. 22) amounts to 9.15 D. This result confirms the pronounced CT behavior of $3^{+3}$ and predicts the existence of dipolar excited states that are responsible for the enhanced $\beta$ values upon protonation. In the same context, the moderate dipole moment change associated with the lowest-energy transitions for $1^{+3}$ and $2^{+3}$ reported in Table VI (3.86 and 4.92 D, respectively) could explain the twofold (fourfold) increase of $\beta_{x x x o}$ in the protonated species $1^{+3}\left(2^{+3}\right)$ with respect to their neutral counterparts 1 (2). Thus, it seems like the contributions of dipolar excited states to the second-order NLO activity increase in the following order: $1^{+3}<2^{+3}<3^{+3}$. Recent studies with three-dimensional chromophores based on substituted

TABLE VI. Results of TD-B3LYP/6-31G** calculations on triprotonated octupolar compounds $1^{+3}$ and $2^{+3}$.

\begin{tabular}{|c|c|c|c|c|c|}
\hline Molecules & $\begin{array}{l}E_{\max } \\
(\mathrm{eV})\end{array}$ & $f_{\text {osc }}{ }^{\mathrm{a}}$ & Major contributions $^{\mathrm{b}}$ & $\begin{array}{c}\left|\mu_{12}\right| \\
\text { (D) }\end{array}$ & $\begin{array}{c}\left|\Delta \mu_{12}\right| \\
\text { (D) }\end{array}$ \\
\hline \multirow{12}{*}{$1^{+3}$} & 3.56 & 1.01 & $\mathrm{H}-1 \rightarrow \mathrm{L}(21 \%)$ & 6.95 & 3.86 \\
\hline & & & $\mathrm{H}-1 \rightarrow \mathrm{L}+1(20 \%)$ & & \\
\hline & & & $\mathrm{H} \rightarrow \mathrm{L}(20 \%)$ & & \\
\hline & & & $\mathrm{H} \rightarrow \mathrm{L}+1(-21 \%)$ & & \\
\hline & 3.56 & 1.01 & $\mathrm{H}-1 \rightarrow \mathrm{L}(-20 \%)$ & 6.95 & 3.86 \\
\hline & & & $\mathrm{H}-1 \rightarrow \mathrm{L}+1(21 \%)$ & & \\
\hline & & & $\mathrm{H} \rightarrow \mathrm{L}(21 \%)$ & & \\
\hline & & & $\mathrm{H} \rightarrow \mathrm{L}+1(20 \%)$ & & \\
\hline & 3.71 & 0.13 & $\mathrm{H} \rightarrow \mathrm{L}+2(87 \%)$ & 2.96 & 12.47 \\
\hline & 3.71 & 0.13 & $\mathrm{H}-1 \rightarrow \mathrm{L}+2(87 \%)$ & 2.96 & 12.47 \\
\hline & 4.10 & 0.25 & $\mathrm{H}-2 \rightarrow \mathrm{L}(91 \%)$ & 3.65 & 11.72 \\
\hline & 4.10 & 0.25 & $\mathrm{H}-2 \rightarrow \mathrm{L}+1(91 \%)$ & 3.65 & 11.72 \\
\hline \multirow[t]{12}{*}{$2^{+3}$} & 3.36 & 0.97 & $\mathrm{H}-1 \rightarrow \mathrm{L}(30 \%)$ & 8.45 & 4.92 \\
\hline & & & $\mathrm{H} \rightarrow \mathrm{L}+1(-30 \%)$ & & \\
\hline & 3.36 & 0.97 & $\mathrm{H}-1 \rightarrow \mathrm{L}+1(30 \%)$ & 8.45 & 4.92 \\
\hline & & & $\mathrm{H} \rightarrow \mathrm{L}(30 \%)$ & & \\
\hline & 3.42 & 0.15 & $\mathrm{H} \rightarrow \mathrm{L}+2(88 \%)$ & 2.92 & 11.73 \\
\hline & 3.42 & 0.15 & $\mathrm{H}-1 \rightarrow \mathrm{L}+2(88 \%)$ & 2.92 & 11.73 \\
\hline & 4.13 & 0.24 & $\mathrm{H}-2 \rightarrow \mathrm{L}(75 \%)$ & 3.45 & 11.10 \\
\hline & 4.13 & 0.24 & $\mathrm{H}-2 \rightarrow \mathrm{L}+1(75 \%)$ & 3.45 & 11.10 \\
\hline & 4.37 & 0.19 & $\mathrm{H}-1 \rightarrow \mathrm{L}+4(36 \%)$ & 2.60 & 9.42 \\
\hline & & & $\mathrm{H} \rightarrow \mathrm{L}+3(36 \%)$ & & \\
\hline & 4.37 & 0.19 & $\mathrm{H}-1 \rightarrow \mathrm{L}+3(36 \%)$ & 2.60 & 9.42 \\
\hline & & & $\mathrm{H} \rightarrow \mathrm{L}+4(-36 \%)$ & & \\
\hline
\end{tabular}

Only transitions with $f_{\text {osc }}>0.10$ are included.

${ }^{\mathrm{b}} \mathrm{H}=\mathrm{HOMO}$, L=LUMO; only contributions above $20 \%$ are included. 
$\left[\mathrm{Ru}^{\mathrm{II}}(\mathrm{bpy})_{3}\right]^{+2}$ derivatives have also elucidated that the electronic transitions to dipolar excited states are responsible for most of the $\beta$ responses in these compounds. ${ }^{27,28}$

\section{CONCLUSIONS}

We reported here a theoretical study of the nonlinear optical properties of newly synthesized pyridine-based octupolar compounds. The calculated $\beta$ values for both neutral and triply charged forms are in good agreement with the experimental data. The relationship between first hyperpolarizability and the chemical pattern modifications (i.e., core substitution, peripheral conjugation) has been also addressed. According to our TD-DFT results, the quadratic NLO response of the neutral octupolar trispyridyl compounds mainly arises from "octupolar contributions" (involving electronic transitions to octupolar excited states which depend on the transition dipole moment between excited states), whereas upon protonation the main contributions to the high $\beta$ values are primarily associated with the electronic transitions to dipolar excited states, which we refer as "dipolar contributions."

\section{ACKNOWLEDGMENTS}

The present work was supported in part by the Dirección General de Enseñanza Superior (DGES, MEC, Spain) through research Project Nos. CTQ2006-14987-C02-01 and CTQ2005-01368. The authors are also indebted to Junta de Andalucía and Gobierno de Aragón (Spain) for funding their research groups FQM-0159 and E39. M.C.R.D. is grateful to the MEC/Fulbright for her Postdoctoral Fellowship at the Georgia Institute of Techology. J.C. is grateful to the MEC of Spain for an I3 professorship position of Chemistry at the University of Málaga and M.M.O. acknowledges the MEC for a personal grant.

${ }^{1}$ J. Zyss, Molecular Nonlinear Optics: Materials, Physics and Devices (Academic, Boston, 1994); Nonlinear Optical Properties of Matter: From Molecules to Condensed Phases, edited by M. G. Papadopoulos, J. Leszczynski, and A. J. Sadlej (Kluwer, Dordrecht, The Netherlands, 2005).

${ }^{2}$ S. R. Marder, C. B. Gorman, F. Meyers, J. W. Perry, G. Bourhill, J. L. Brédas, and B. M. Pierce, Science 265, 632 (1994); S. R. Marder, Chem.
Commun. (Cambridge) 2006, 131; H. Kang, G. Evmenenko, P. Dutta, K. Clays, K. Song, and T. J. Marks, J. Am. Chem. Soc. 128, 6194 (2006); M. Yang and B. Champagne, J. Phys. Chem. A 107, 3492 (2003); Y. Liao, S. Bhattacharjee, K. A. Firestone, B. E. Eichinger, R. Paranji, C. A. Anderson, B. H. Robinson, P. J. Reid, and L. R. Dalton, J. Am. Chem. Soc. 128, 6847 (2006); H. Kang, A. Facchetti, H. Jiang, E. Cariati, S. Righetto, R. Ugo, C. Zuccaccia, A. Macchioni, C. L. Stern, Z. Liu, S.-T. Ho, E. C. Brown, M. A. Ratner, and T. J. Marks, ibid. 129, 3267 (2007). ${ }^{3}$ J. Zyss and I. Ledoux, Chem. Rev. (Washington, D.C.) 94, 77 (1994).

${ }^{4}$ B. J. Coe, L. A. Jones, J. A. Harrs, B. S. Brunschwig, I. Asselberghs, K. Clays, A. Persoons, J. Garin, and J. Orduna, J. Am. Chem. Soc. 126, 3880 (2004)

${ }^{5}$ G. Hennrich, I. Asselberghs, K. Clays, and A. Persoons, J. Org. Chem. 69, 5077 (2004).

${ }^{6}$ I. Asselberghs, K. Clays, A. Persoons, M. D. Ward, and J. McCleverty, J. Mater. Chem. 14, 2831 (2004).

${ }^{7}$ I. Ratera, C. Ruiz-Molina, S. Sporer, S. Marcen, S. Montant, J. F. Letard, E. Freysz, C. Rovira, and J. Veciana, Polyhedron 22, 1851 (2006).

${ }^{8}$ P. C. Ray and J. Leszczynski, Chem. Phys. Lett. 399, 162 (2004).

${ }^{9}$ I. Asselberghs, G. Hennrich, and K. Clays, J. Phys. Chem. A 110, 6271 (2006).

${ }^{10}$ M. J. Frisch et al., GAUSSIAN 03, Revision B.05, Gaussian, Inc., Pittsburgh, PA, 2003.

${ }^{11}$ A. D. Becke, J. Chem. Phys. 98, 1372 (1993).

${ }^{12}$ M. M. Francl, W. J. Pietro, W. J. Hehre, J. S. Binkley, M. S. Gordon, D. J. Defrees, and J. A. Pople, J. Chem. Phys. 77, 3654 (1982).

${ }^{13}$ E. Runge and E. K. U. Gross, Phys. Rev. Lett. 52, 997 (1984).

${ }^{14}$ W. Koch and M. C. Holthausen, A Chemist's Guide to Density Functional Theory (Wiley, Weinheim, 2000).

${ }^{15}$ S. Portmann and H. P. Lüthi, Chimia 54, 766 (2000).

${ }^{16}$ P. Pulay, J. Chem. Phys. 78, 5043 (1983).

${ }^{17}$ C. E. Dykstra and P. G. Jasien, Chem. Phys. Lett. 109, 388 (1984).

${ }^{18}$ J. Zyss, Nonlinear Opt. 1, 3 (1991).

${ }^{19}$ J. Zyss, Chem. Phys. 98, 6583 (1993).

${ }^{20}$ M. Joffre, D. Yaron, R. J. Silbey, and J. Zyss, J. Chem. Phys. 97, 5607 (1992).

${ }^{21}$ W. Zhu and G. S. Wu, J. Phys. Chem. A 105, 9568 (2001).

${ }^{22}$ M. Moreno, J. T. Lopez Navarrete, J. Casado, G. Hennrich, M. C. Ruiz Delgado, and J. Orduna, "Linear and non-linear optical properties of pyridine-based octopolar chromophores designed for chemical sensing: Joint spectroscopic and theoretical study," J. Phys. Chem. C. (to be published).

${ }^{23}$ C. Andraud, T. Zabulon, A. Collet, and J. Zyss, J. Chem. Phys. 245, 243 (1999).

${ }^{24}$ Y. K. Lee, S. J. Jeon, and M. Cho, J. Am. Chem. Soc. 120, 10921 (1998).

${ }^{25}$ W. H. Lee, H. Lee, J. A. Kim, J. H. Choi, M. Cho, S. J. Jeon, and B. R. Cho, J. Am. Chem. Soc. 123, 10658 (2001).

${ }^{26}$ M. Cho, H. S. Kim, and S. J. Jeon, Chem. Phys. 108, 7114 (1998).

${ }^{27}$ F. W. Vance and J. T. Hupp, J. Am. Chem. Soc. 121, 4047 (1999).

${ }^{28}$ B. J. Coe, J. A. Harris, B. S. Brunschwig, I. Asselberghs, K. Clays, J. Garin, and J. Orduna, J. Am. Chem. Soc. 127, 13399 (2005). 